\title{
Capacidad innovadora y crecimiento regional en México: un enfoque espacial
}

\section{Innovative capacity and regional growth in Mexico: a spatial approach}

\author{
Jesús Armando Ríos-Flores* \\ Juan Manuel OceguedaHernández*
}

\begin{abstract}
This study presents an analysis of the effect of innovative capacity on economic growth under a time-space framework. Recognizing the limitations of the representativeness of intellectual property in the institutionally weak regions, an aggregate indicator for innovative capacity is generated using a factorial model and thus compare the different effects of both indicators. The results show indicates that the ability to innovate, in the case of patents, is only significant in the presence of spatial effects, while the aggregate indicator is significant in all cases, being greater when the spatial effect is relevant.
\end{abstract}

Keywords: innovative capacity, economic growth, factorial model, spatial econometrics.

\section{Resumen}

Este estudio presenta un análisis sobre el efecto de la capacidad innovadora en el crecimiento económico bajo un marco espacio-temporal. Al reconocer las limitantes de la representatividad de la propiedad intelectual en las regiones institucionalmente débiles, se genera un indicador agregado para la capacidad innovadora mediante un modelo factorial, que permita comparar los efectos de ambos indicadores. Los resultados muestran que la capacidad para innovar, en el caso de las patentes, sólo es significativa en presencia de efectos espaciales, mientras el indicador agregado es significativo en todos los casos, y es aún mayor cuando el efecto espacial es relevante.

Palabras clave: capacidad innovadora, crecimiento económico, modelo factorial, econometría espacial.

*Universidad Autónoma de Baja California, correos-e: jrios89@uabc.edu.mx, jmocegueda@ uabc.edu.mx 


\section{Introducción}

En una economía dinámica, globalizada y competitiva la innovación se coloca como la estrategia central para el incremento de la cuota de mercado y el crecimiento económico (Fagerberg et al., 2010). La mayoría de los países que han visto en la innovación una oportunidad para el crecimiento han generado estímulos financieros para el desarrollo científico y tecnológico, mientras que para potencializar su efecto se han desarrollado estrategias de vinculación para la generación de redes que permitan incrementar la capacidad para innovar mediante la acumulación y los spillovers del conocimiento (Coe y Helpman, 1995; Metcalfe, 2002). En este sentido, habría que cuestionarse ¿qué efecto ha generado la Capacidad Innovadora (CI) en el crecimiento económico en México? y ¿cómo han influido las agrupaciones geográficas tanto en la innovación como en el crecimiento?

En los estudios empíricos del crecimiento vía innovación para México, en la mayor parte de los casos, se recurre a los indicadores de patentes o al gasto en investigación y desarrollo (GIDE) (Hernández y Díaz, 2007; Mendoza et al., 2008; Guzmán et al., 2008; Villareal, 2012), variables un tanto limitadas en el contexto de un país institucionalmente débil (Dosi et al. 2006; Ríos y Castillo, 2015). Por una parte el contenido tecnológico en los instrumentos de propiedad intelectual es diverso (Jaffe y Trajtenberg, 2002), y dado el efecto temporal en la garantía de los derechos genera que la propiedad intelectual contenga un efecto estratégico en cuanto a su solicitud (Albornoz, 2009; Ríos y Castillo, 2013), por lo que no necesariamente reflejan la CI o la intención de sacar un nuevo producto al mercado.

De forma adicional, en el caso de México, gran parte de las solicitudes de patentes provienen de los organismos del sector público por lo que no reflejan fehacientemente la capacidad tecnológica de la economía regional (López, 2008). Para abordar el estudio económico de la innovación, sin limitarse a la estadística del sistema de patentes o al GIDE, Torres-Preciado et al. (2014) presentan un indicador agregado mediante la agrupación de instrumentos de la propiedad intelectual. Por su parte, Casas (2001), Ruiz (2008), Sánchez et al. (2014) y Valdez-Lafarga y LeónBalderrama (2015) construyen otro con una variedad de factores como los recursos para la innovación, las estructuras productivas y las cuestiones institucionales, intentando representar la dinámica del Sistema Regional de Innovación (SRI) y no sólo algunos de sus resultados. En estos últimos casos, que intentan representar al SRI, sus aportaciones se limitan a un sólo periodo y sus relaciones causales no contemplan de forma explícita 
el efecto espacial. Si bien ya empiezan a sumarse un mayor número de estudios sobre la innovación, la escasez de información temática, espacial y temporal los mantienen restringidos en su mayoría al caso de las solicitudes de patente.

En las regiones, al interior de un país, existen espacios heterogéneos en términos productivos e institucionales (Aroca et al., 2005), y la movilidad de los factores se genera de manera más ágil debido a la inexistencia de barreras proteccionistas (Gluckler, 2007). Estos efectos estructurales han sido ampliamente tratados en los estudios de convergencia en la teoría del crecimiento (Sala-i-Martín, 2000), pero en la mayor parte de los casos omiten el efecto de la interdependencia espacial, el cual puede generar un proceso endógeno en el espacio (Rey y Mounturi, 1999). Los estudios que abordan de forma explícita el efecto de interacción espacial de la innovación o el crecimiento regional en México son limitados. Valdivia (2007) y Torres-Preciado et al. (2014) presentan evidencia de efectos espaciales del conocimiento tecnológico en el crecimiento regional, y otros, como Aroca et al. (2005), Rey y Sastré (2010) y Sastré y Rey (2013) presentan evidencia de un proceso de divergencia regional en el ingreso, intensificándose a partir de la mayor integración con el mercado internacional.

El objetivo de este estudio es estimar el efecto de la CI en el crecimiento económico. Por una parte se prueba con el indicador tradicional de patentes y, por otro, con un indicador agregado de capacidad innovadora, el cual refleja la capacidad de producción, adquisición y asimilación tecnológica, reflejo de las políticas e inversiones que sustentan el proceso innovador. En un segundo aspecto y, reconociendo el posible efecto geográfico, se presenta una función de tipo tiempo-espacio, para las entidades federativas de México tomando dos periodos temporales, 1998-2003 y 2004-2008, posteriores a la apertura comercial y previos a la crisis de 2008 , en los cuales se presenta una primera reestructuración de la economía mexicana ante la globalización. El indicador agregado se genera mediante la selección de un conjunto de variables tecnológicas, cuya reducción de la dimensión se realiza con un modelo factorial, y los efectos geográficos se abordan mediante la metodología de la econometría espacial.

La estructura del documento consta de cuatro apartados, en el primero se presenta una revisión teórica sobre los efectos de la innovación en el crecimiento económico y sobre los efectos inter-regionales; en el segundo se expone la metodología, en la que se refieren las ecuaciones a estimar bajo el marco general de la econometría espacial, asimismo, se advierte sobre la generación del indicador agregado de la CI, así como la estructura de los datos. En el tercer apartado se presentan los resultados, en el 
cual se comparan los efectos tanto de la variable patentes como del indicador agregado de capacidad innovadora en ambos periodos. En el cuarto apartado se presentan las conclusiones.

\section{Innovación, crecimiento y efectos regionales}

En la visión de Solow (1957), como eje de la teoría neoclásica del crecimiento vía innovación, un primer aspecto a destacar es el predominio del cambio técnico como un fenómeno exógeno. La superación de esta visión ha puesto énfasis en el vehículo del cambio tecnológico. Romer (1990), Grossman y Helpman (1991) y Aghion y Howitts (1992) desplazan el énfasis de la formación de capital fijo a la de capital humano y conocimiento tecnológico, enfocándose en los factores organizacionales y de aprendizaje en el crecimiento, suponiendo la acumulación de capital físico como respuesta a la acumulación de conocimiento. De forma adicional, Nelson y Winter (1982) agregan que el proceso innovador es interactivo, ya que se encuentra signado en la incertidumbre y está sujeto a externalidades y rendimientos dinámicos.

En términos generales la innovación depende del conocimiento científico y su interacción social (López-Leyva et al., 2014), su origen puede ser interno o externo (Archibugui y Michie, 1997) y estar incluida en bienes de capital (RICYT, 2001) o en el saber hacer de las empresas y sus trabajadores (Arrow, 1962). Parte importante de la actividad innovadora se encuentra en la estructura de las firmas (Pavitt, 1984), las redes empresariales (Mungaray y Palacio, 2000) y una variedad de organizaciones e instituciones que delimitan la interacción económica (Nelson, 1994; Lundvall, 1992; Cooke, 1992). En las regiones, las estructuras y componentes de un sistema de innovación son significativamente diferentes y, por tanto, las dinámicas e interacciones de las firmas con su entorno institucional determinan sus características y efectos (Autio et al. 2014; Ríos y Castillo, 2015).

La tecnología no es un bien disponible sin costo, requiere de una acumulación de conocimiento específico, adquirido a través del proceso de aprendizaje y determinado por ese mismo conocimiento (Dosi, 1988). Parte del conocimiento tecnológico es idiosincrásico, a menudo tácito y de adquisición costosa, por lo que no todas las empresas tendrán los mismos resultados por tener acceso a la misma información, lo que acentúa la formación de capacidades adquiridas y la trayectoria tecnológica en el proceso de aprendizaje (Lall, 1992; Krammer y Sorin, 2009). En esta perspectiva, el empresario y su interacción con el entorno toman un papel protagónico al determinar el proceso innovador (Autio et al. 2014). 
$\mathrm{Al}$ reconocer las diferencias estructurales e institucionales en las regiones de México, Ruiz (2008), Sánchez et al. (2014) y Valdez-Lafarga y León-Balderrama (2015) generan una tipología del SRI, enfatizando la importancia del sistema empresarial, institucional y educativo como los factores de mayor influencia para la CI. Dada las particularidades y la marcada diferencia entre entidades, el SRI materializa el espacio idóneo para el aprendizaje colectivo y la transferencia de conocimiento tácito.

La evidencia internacional ha mostrado un estancamiento en innovación en los países no desarrollados ya que el incremento en el GIDE no ha generado los resultados de innovación deseados (Aboites y Soria, 2008; Dosi et al., 2006). De forma similar, las tecnologías que trascienden en nuevos productos y procesos en el mercado suelen ser de los países desarrollados (Furman et al., 2002). En el ámbito regional se presenta un caso muy similar. La explicación reside en que las empresas líderes, geográficamente localizadas, conscientes en su ventaja competitiva no tratan de expulsar a las competidoras para quedarse con el mercado compitiendo con base en los precios (Baldwin y Okubo, 2006). Buscan maximizar beneficios a largo plazo, a base de asegurar la plena utilización de su capacidad productiva y de mantener o ampliar su poder de mercado por medio de la innovación, lo que genera la concentración.

Una vez que se constata que el desarrollo de una región se caracteriza por su estructura y trayectoria económica (intra-regional), se realiza un análisis de dependencia espacial (inter-regional) que tiene sus fundamentos en bases geográficas (Krugman, 1998). En este sentido, cualquier explicación sobre dinámicas regionales debe considerar la interdependencia que surge por la ubicación geográfica y el entorno regional en que se encuentra (Moran, 1948; Butler, 1994). Dentro de este enfoque surgen nociones que destacan la idea de externalidades positivas (Coe y Helpman, 1995) y economías de aglomeración (Porter, 1990) al hecho de que una fuente importante del mejoramiento en la productividad viene dado por condiciones espaciales en un efecto spillover geográficamente mediatizado (Krugman, 1991, Fujita y Krugman, 2004).

Lall (1992) explica que las capacidades tecnológicas desempeñan un doble papel: son mecanismos de asimilación, pero a la vez, pueden crear las condiciones para pasar de la etapa de asimiladores a la de productores de nuevo conocimiento. Las regiones innovadoras se caracterizan por su dinámica productiva sustentada en los sectores en alta tecnología, dependientes del conocimiento interno, mientras el resto de regiones presentan dinámicas productivas diversificadas con implementación del conocimiento principalmente externo (Archibugui y Michie, 1997). En las regiones con crecimiento vía proximidad, la característica principal es la imitación y la dependencia tecnológica del espacio contiguo (Rozga, 
2008). Si bien la difusión del conocimiento es incontrolable y los canales de difusión son formalmente los mismos, la transferencia de conocimiento no es homogénea, ni mantiene los mismos patrones ya que requieren de una capacidad para absorber las nuevas tecnologías y la capacidad industrial para su posterior implementación en la actividad económica local (Omidvar, 2013).

$\mathrm{Al}$ igual que en el entorno internacional, en el local, surgen efectos de crecimiento desequilibrado donde aparecen regiones que se desarrollan y otras que se rezagan (Kaldor, 1970; Fujita y Krugman, 2004). En ambos niveles surgen externalidades positivas (difusión) y negativas (polarización). Los efectos de difusión consisten en el incremento en las compras y las inversiones en las regiones periféricas o mediante la absorción de parte de su desocupación disfrazada, aumentando la productividad marginal y los niveles de consumo per cápita de éstos. Los efectos de polarización consisten en la concentración del progreso económico, ya que las actividades con mayor productividad marginal se encuentran en los lugares centrales donde se concentran las oportunidades de desarrollo (Myrdal, 1957; Baldwin y Okubo, 2006). En este caso, la convergencia económica surgirá, si y sólo si, el efecto de difusión es mayor que el de polarización, tanto para el caso internacional como local (Hirschman, 1958).

La imitación, como medio de convergencia tecnológica, no sólo es más frecuente que las actividades de innovación, sino que es una de las vías predominantes en la estandarización productiva, y estímulo al crecimiento de las empresas y sus beneficios (Mansfield, 1961; Lundvall, 1992 y Lall, 1992). La imitación es el mecanismo más poderoso de la difusión del nuevo conocimiento (Metcalfe, 2002), sin lo cual el conocimiento producido, que da lugar a nuevos mercados, no tendría repercusiones económicas (Vázquez-Barquero, 2005). Sin embargo, desde la perspectiva del productor de conocimiento estas características se convierten en incertidumbre en la apropiación de los beneficios derivados del esfuerzo inventivo y pueden erosionar las bases de la producción de nuevo conocimiento (Oster, 2000). Así, los derechos de propiedad intelectual se colocan como un mecanismo que media entre el estímulo a la innovación y la imitación productiva (Gould y Gruben, 1995).

En la geografía económica, los SRI como un lugar o un sistema de lugares, la contigüidad territorial desempeña un papel clave en la homogeneidad cultural regional y la facilidad de circulación de conocimiento (Gluckler, 2007). En este sentido, la innovación presenta una diversidad de matices ligadas a los efectos estructurales, espaciales y temporales (Hall y Rosenberg, 2010). En los lugares centrales donde se realiza la innovación se presentan efectos directos en el crecimiento económico de su propio desarrollo tecnológico, mientras que en el resto de lugares, llamémoslos 
dependientes o imitadores, dependen del avance tecnológico de los centrales cuyo éxito en la implementación depende de su estructura productiva y su capacidad de aprendizaje (Solo, 1966).

Mansfield (1961) y Griliches (1960), para el caso de Estados Unidos, generan evidencia de que el crecimiento de las regiones vía innovación va acompañada muy de cerca por el efecto de la imitación y el aprendizaje con un comportamiento de tipo logístico. Dentro de los primeros trabajos en utilizar la idea del espacio como fenómeno a medir se encuentran los de Jaffe (1989) y Anselin et al. (1997). Estos estudios, en línea a lo propuesto por Griliches, agregan la existencia de spillovers geográficamente mediatizados, con objeto de analizar la importancia de la proximidad geográfica en la captura y aprovechamiento de externalidades tecnológicas. Encuentran que estos indicadores son estadísticamente significativos y que los efectos de la innovación decrecen con la distancia al centro donde se originan.

Al retomar los efectos espaciales de la producción y difusión del conocimiento, así como de sus impactos económicos, Anselin et al. (2000), Acs et al. (2002) y Fisher y Varga (2003) presentan evidencia de que la difusión espacial del conocimiento es una función decreciente con la distancia y positiva con GIDE. Este tipo de modelos espaciales son más bien la excepción a la regla en las investigaciones empíricas de la tecnología. En el caso de estudios sobre difusión de las innovaciones y el crecimiento regional en México su existencia es limitada. Valdivia (2007) y Torres-Preciado et al. (2014) presentan evidencia de efectos espaciales del conocimiento tecnológico en el crecimiento regional, mostrando que las entidades federativas como la Ciudad de México, Nuevo León y Jalisco presentan efectos de arrastre tecnológico sobre sus regiones más próximas, y concuerdan con Aroca et al. (2005), Rey y Sastré (2010) y Sastré y Rey (2013) en que el país vive un proceso de divergencia entre regiones desde la integración comercial con América del norte.

\section{Capacidad innovadora y efecto espacial}

\subsection{Modelo y método de estimación}

En el caso de los estudios regionales en México, mediante los indicadores de la propiedad intelectual y el GIDE, Mendoza et al. (2008), Marroquín y Ríos (2012), Ríos y Marroquín (2013) encuentran una relación positiva de la innovación con el crecimiento económico. Una de las cuestiones ligadas a la contigüidad territorial y la libre movilidad de los factores, dada la multidireccionalidad que domina la relación entre unidades espaciales 
vecinas, es la dependencia espacial en un posible proceso endógeno territorial.

En el caso de Sánchez et al. (2014) abordan la innovación desde un enfoque agregado, sin tomar en cuenta el espacio; por su parte, TorresPreciado et al. (2014) abordan de forma explícita el efecto espacial, pero se limitan a los indicadores de la propiedad intelectual. Para resolver esta diversidad de problemas de medición y determinar el efecto de la capacidad innovadora se genera un indicador agregado mediante la conjunción de una variedad de indicadores que serán simplificados a través de un modelo factorial, mientras que la cuestión del efecto sobre el ingreso se utiliza el análisis econométrico espacial en una función dinámica, de la forma siguiente:

$$
\begin{gathered}
P I B_{i t}=\beta_{0}+\beta_{1} P I B_{i t-1}+\beta_{2} W P I B_{j t}+\beta_{3} p_{i t}+(\lambda W e+u) \\
P I B_{i t}=\beta_{0}+\beta_{1} P I B_{i t-1}+\beta_{2} W P I B_{j t}+\beta_{3} C l_{i t-1}+(\lambda W e+u)
\end{gathered}
$$

Donde $P I B$ representa el producto interno bruto per cápita en el momento $t$ para la entidad federativa $i$. PAT representa las solicitudes de patente por cada 10000 habitantes, $C I$ representa el indicador agregado de la capacidad innovadora, WPIB representa el efecto de la dependencia espacial sustantiva dada la vecindad territorial, la cual es obtenida mediante la matriz de pesos espaciales $W$. En el caso de el efecto espacial residual y $u$ representa el error aleatorio, mientras representa la elasticidad para cada una de las variables. Para el caso de las variables estructurales se utiliza el PIB rezagado temporal y espacialmente, que engloban todo el conjunto de efectos estructurales para obtener el efecto de la innovación lo más robusto posible, la cual se estimará bajo el marco de métodos espaciales.

El efecto de dependencia espacial es una relación funcional entre una unidad espacial y otras que son cercanas a ésta (Cliff y Ord, 1981; Anselin, 1988). Consiste en la ausencia de independencia en observaciones de conjuntos de datos transversales geo-referenciados, lo cual implica que el valor de una variable está condicionado por el valor de esa variable en una región vecina. Si el fenómeno en una región se extiende hacia el resto de las ubicaciones que la rodean y dicha expansión genera concentración, se trata de un caso de autocorrelación positiva. En esta situación existe asociación entre valores similares de una variable y localizaciones cercanas, donde se presentan valores altos (bajos) rodeados de valores altos (bajos) en un efecto tipo desbordamiento o spillover. Por el contrario, existe autocorrelación espacial negativa cuando la manifestación de un fenómeno en una unidad obstaculiza su aparición en las unidades vecinas. El escenario de este caso se halla dominado por valores altos rodeados de valores bajos y 
viceversa, donde existe una jerarquía de tipo "centro-periferia" (Moreno y Vayá, 2002).

La herramienta principal para el análisis geo-referenciado es la matriz de pesos espaciales $(W)$, la cual captura cualquier tipo de relación geográfica (LeSage y Pace, 2009). Siendo $w$ una matriz cuadrada de tamaño NXN (donde $\mathrm{N}$ son las unidades espaciales), no estocástica cuyos elementos $w_{i j}$ reflejan la intensidad de la interdependencia entre cada par de regiones $i$ y $j$ (Anselin, 1988). La forma general en que puede ser definida es una matriz de contigüidad binaria, en la que el valor de cada $w_{i j}$ se basa en la determinación de adyacencia. De forma simplificada $w_{i j}=1$ si ambas unidades espaciales comparten una frontera de longitud no nula y $w_{i j}=0$ en caso contrario.

En la literatura se proponen numerosos estadísticos para el descubrimiento de relaciones de interacción o asociación espacial, siendo el de uso generalizado el I de Moran (Moreno y Vayá, 2002) para el caso de dependencia espacial global, bajo la forma:

$$
I=\frac{N}{s_{0}} \frac{\sum_{i j}^{N} w_{i j}\left(x_{i}-\bar{x}\right)\left(x_{j}-\bar{x}\right)}{\sum_{i-1}^{N}\left(x_{i}-\bar{x}\right)^{2}}
$$

Donde $i \neq j$, $x_{i}$ es el valor de la variable cuantitativa $x$ en la región $i$, x es la media muestral de $x, W_{i f}$ son los pesos de la matriz W, N, es el tamaño muestral, $\mathrm{S}_{0}=\sum i \sum j$ wij es la distancia máxima a la que se encuentra la unidad $j$ de la $i$ para considerarse vecinas y $z$ es el valor de contraste de una tabla normal estándar. El I de Moran toma valores entre el intervalo $[-1,1]$, donde los extremos representa una perfecta asociación espacial. Si el fenómeno en una región se extiende hacia el resto de las ubicaciones que la rodean y dicha expansión genera concentración, se trata de un caso de autocorrelación positiva. Por el contrario, existe autocorrelación espacial negativa cuando la manifestación de un fenómeno en una unidad obstaculiza su aparición en las unidades vecinas (LeSage y Pace, 2009).

El supuesto estadístico I de Moran se basa en que, bajo una muestra grande, las variables se normalizan bajo una distribución normal asintótica. En el caso de las muestras pequeñas es necesario un enfoque basado en la permutación espacial estocástica para la normalización de variables (Rey, 2000).

Un instrumento gráfico basado en el I de Moran es el scatterplot, el cual se representa en un plano cartesiano como el que describe la figura 1, con las observaciones de la variable de interés normalizada de la región i en el eje de las abscisas y el retardo espacial de la misma en el eje de las ordenadas, donde el arreglo de los puntos en los cuatro cuadrantes indica el 


\section{Figura 1}

\section{Perfil del espacio del scatterplot de Moran}

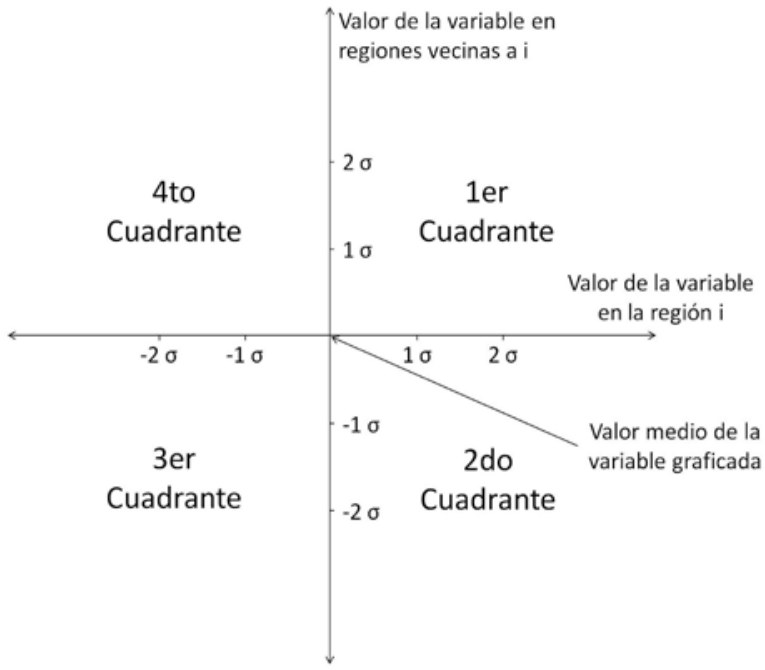

Fuente: Basado en Moreno y Vayá (2000).

tipo de asociación espacial presente. En el cuadrante 1 se presentan los individuos de valores altos con vecinos altos, en e l cuadrante 3 se presentan los individuos de valores bajos con vecinos bajos, mientras en los cuadrantes 2 se presentan los individuos de valores altos con vecinos bajos y en el cuadrante 4 es a la inversa.

Si los puntos están ordenados en los cuadrantes 1 y 3 indican que existe dependencia espacial positiva: las regiones con niveles superiores/ inferiores a la media están rodeadas de otras semejantes; mientras que cuando los valores se concentran en los cuadrantes restantes, la dependencia espacial que existe es negativa: las regiones con niveles mayores/ inferiores a la media se hallan cerca de otras con niveles opuestos. Si los puntos no siguen un patrón y se encuentran dispersos en el espacio, se presume ausencia de dependencia espacial (Moreno y Vayá, 2000).

Los contrastes de autocorrelación global poseen la limitación de no capturar el efecto particular de dependencia, ya que el esquema detectado pudiera no cumplirse para todas las unidades del espacio analizado (Anselin, 1995). Debido a esto, se presentan los indicadores locales de asociación espacial (LISA) para verificar la existencia de clusters regionales, bajo la forma:

$$
L I S A_{i}=\frac{z_{i}}{\sum_{i} z_{i} / N} \sum_{j \epsilon i j_{i}} w_{i j} z_{j}
$$


Donde $\mathrm{z}_{i}$ es el valor de la región $i$ de la variable normalizada y $J_{i}$ el conjunto de regiones vecinas a $i$. Con signo positivo se presenta el caso de clusters de valores similares alrededor de la región $i$ y viceversa en caso negativo.

El indicador local de asociación espacial permite descomponer el indicador de asociación global en la contribución marginal de cada observación en particular, evaluando la significancia del agrupamiento alrededor de una observación e indicar el grado de heterogeneidad espacial presente en la muestra. LISA presenta cuatro tipos de cluster regionales: 1. high-high, donde se presenta un tipo de asociación regional de entidades con valores altos rodeados de vecinos similares; 2. low-high, donde las entidades con valores altos se encuentran alrededor de entidades de valores bajos; 3 . highlow, de valores bajos rodeados de altos y; 4 . low-low, donde se concentran entidades de regiones con indicadores bajos.

De acuerdo con Anselin (1995), LISA proporciona una cuantificación del grado de agrupamiento significativo de valores similares alrededor de una observación, mientras la suma de los LISA es proporcional a un indicador global de asociación espacial. Dado que los indicadores globales y regionales son similares, la presencia de un indicador global positivo es congruente con clusters high-high y low-low, mientras que un indicador global negativo es congruente con clusters high-low y low-high.

Existen diferentes contrastes para detectar la presencia de dependencia espacial, además de la posibilidad de detectar su tipología. El modelo a seleccionar se determina por dos factores asociados a la dependencia espacial. En primer lugar, es posible que exista autocorrelación espacial sustantiva como consecuencia de la existencia de variables sistemáticas, endógenas o exógenas correlacionadas espacialmente. En segundo lugar, el efecto de autocorrelación espacial residual puede estar presente como consecuencia de la existencia de un esquema de dependencia espacial en el término de perturbación (Moreno y Vayá, 2000). La especificación espacial se define bajo la forma general siguiente:

$$
\begin{gathered}
y=\rho W y+\beta X+\delta W X+\varepsilon \\
\varepsilon=\lambda W \varepsilon+u
\end{gathered}
$$

Donde $y$ es un vector $N x 1$ de las $N$ observaciones, $\rho$ es el parámetro autorregresivo que recoge la intensidad de las interdependencias entre las observaciones muestrales, $W y$ es el retardo espacial de la variable dependiente, $\beta$ representa el impacto de la variable exógena sobre la variable dependiente, es una matriz de $k$ variables exógenas, $W K$ es el retardo espacial de las variables exógenas, $\delta$ captura el efecto de la(s) variable(s) independiente(s) sobre la variable dependiente de sus vecinos y un tér- 
mino de perturbación aleatoria. $\mathrm{Al}$ imponer restricciones a los parámetros $\delta$ y $\lambda$ se obtienen cuatro casos particulares: el modelo con rezago espacial en la variable dependiente (SAR), el modelo con rezago espacial en el término de error (SEM), uno especial que considera ambos casos (SARAR) (Moreno y Vayá, 2000).

El modelo SAR es un caso particular cuando que al ser reemplazando en la ecuación espacial general se obtiene, en el cual la relación espacial se produce directamente por la presencia de la variable endógena rezagada, mientras que no considera la relación directa entre las características propias de las vecinas con la variable endógena. Por otra parte, el modelo SEM supone, de modo que, donde. En este modelo, la relación espacial se produce por el denominado efecto contagio, donde los choques exógenos se propagan entre las unidades vecinas.

En el caso del modelo SARAR que se representa con rezago espacial en la variable dependiente y en el término de error asume un se obtiene:

$$
\begin{gathered}
y=\rho W y+\beta X+\varepsilon \\
\varepsilon=\lambda W \varepsilon+u
\end{gathered}
$$

El supuesto del modelo SARAR es que la relación espacial se presenta por dos mecanismos, el primero por medio de la relación directa entre la variable endógena y su rezago espacial. El segundo se produce por choques aleatorios en las unidades vecinas, las cuales tienen un efecto contagio sobre la unidad $i$.

Para confirmar la presencia del efecto de dependencia son habituales los contrastes basados en los multiplicadores de Lagrange como el test $L M_{\text {lag }}$ propuesto por Anselin (1988) y el test $L M_{l e}$ de Bera y Yoon (1992), bajo la hipótesis nula de $\rho=0$. Cuando la dependencia espacial es referida al término de error se utilizan los contrastes $L M_{e r r}$ (Burridge, 1980) y su versión robusta $L M_{l e}$ (Bera y Yoon, 1992), además del contraste I de Moran, bajo la hipótesis nula de $\lambda=0$. Finalmente, para contrastar la existencia conjunta de ambos tipos de dependencia espacial, se utiliza el test SARMA, con las hipótesis nulas de $\rho, \lambda=0$. La decisión estadística sobre la especificación del modelo se basa en los contrastes de hipótesis que evalúan la relación con las opciones derivadas del modelo espacial general, las cuales son estimadas en el software estadístico Space.

\subsection{Construcción de indicadores}

Dentro de las dificultades referentes a la medición de la CI es determinar cuando la CI de un agente es superior a la de otro, dado que las estruc- 
turas productivas son cuantitativa y cualitativamente diferentes. Por una parte, se podría argumentar que los agentes que presentan mayores beneficios ligados a los productos innovadores son los que presentan una mayor $\mathrm{CI}$, pero existe evidencia de que una parte importante de las innovaciones en el mercado surgen de una serie de eventos no intencionados. Por otra parte, el hecho de poseer capacidades tecnológicas desarrolladas, tanto en el monto como en el personal en investigación y desarrollo, no garantizan que los esfuerzos se traducirán en productos exitosos. Para resolver este problema, la construcción del indicador de la CI incluye variables tecnológicas de recursos, resultados y de estructura de apoyo y soporte para intentar captar de forma envolvente el sRI. En el cuadro 1 se presentan los indicadores que se sujetan al Modelo Factorial (MF) para la construccióde

\section{Cuadro 1}

\section{Indicadores utilizados para la construcción de la CI}

\begin{tabular}{lll}
\hline \multicolumn{1}{c}{ Variables } & clave & Fuente \\
\hline $\begin{array}{l}\text { Patentes solicitadas por cada } \\
10,000 \text { habitantes }\end{array}$ & PAT & Conacyt \\
$\begin{array}{l}\text { Valor agregado censal bruto por } \\
\text { habitante en las industrias de alta } \\
\text { tecnología }\end{array}$ & VH & Inegi \\
$\begin{array}{l}\text { Miembros del sistema nacional de } \\
\text { investigadores por centro de in- } \\
\text { vestigación }\end{array}$ & ICI & Conacyt, Cofupro y FCCYT \\
$\begin{array}{l}\text { Personal ocupado en las industrias } \\
\text { de alta tecnología por cada 1000 } \\
\text { habitantes }\end{array}$ & EH & \\
$\begin{array}{l}\text { Unidades económicas en las in- } \\
\text { dustrias de alta tecnología por } \\
\text { cada } 10,000 \text { habitantes }\end{array}$ & UE & Inegi \\
$\begin{array}{l}\text { Empresas con certificado ISO } \\
\text { 9000 por cada 10,000 habitantes } \\
\begin{array}{l}\text { Número de contratos en telefonía } \\
\text { móvil por cada 100 habitantes }\end{array}\end{array}$ & EC & Inegi \\
$\begin{array}{l}\text { Número de teléfonos fijos por } \\
\text { cada 100 habitantes }\end{array}$ & TEL & Conacyt \\
$\begin{array}{l}\text { Activo total por habitante en las } \\
\text { industrias de alta tecnología }\end{array}$ & ATH & Cofetel \\
\hline
\end{tabular}

Fuente: elaboración propia. Conacyt: Consejo Nacional de Ciencia y Tecnología; Inegi: Instituto Nacional de Estadística y Geografía; Cofupro: Consejo de Fundaciones Produce; FCCYT: Foro Consultivo, Científico y Tecnológico; Cofetel: Comisión Federal de Telecomunicaciones. La clasificación tecnológica es similar a Ocegueda et al. (2009). 
la CI siendo ajustados en todos los casos en términos de la población con datos del Consejo Nacional de Población.

El propósito del mF es encontrar el número mínimo de dimensiones a partir de las cuales se explique el máximo de información generada en una investigación, simplificando las múltiples y complejas relaciones entre un conjunto de variables observables . En particular, se trata de encontrar factores comunes que expliquen de modo suficiente las variables originales observables sin presentar juicios a priori sobre el peso que debe tener cada variable en los factores y de cada factor en la CI (Pérez, 2006).

La aplicación del MF requiere desarrollar cuatro fases interdependientes: 1. el cálculo de una matriz que indique la variabilidad de los indicadores estudiados; 2. la extracción de los factores que agrupan a un conjunto de variables con características comunes; 3 . la rotación de la matriz de componentes principales y; 4 la estimación de las puntuaciones de las variables en los factores (Pérez, 2006). Los cuales se resumen en cuadro 2 para el MF.

El paso final del MF es el de estimar las puntuaciones de las variables en las componentes. Cada factor es ponderado por las puntuaciones de

Cuadro 2

El análisis factorial

\begin{tabular}{cccc}
\hline Características & \multicolumn{3}{c}{ Factores } \\
\cline { 2 - 4 } & vh $(.914)$ & PAT $(.819)$ & EC $(.745)$ \\
\hline & ATH $(.692$ & UE $(.85$ I $)$ & TEL $(.873)$ \\
& EH $(.760)$ & ICI $(.823)$ & CEL $(.885)$ \\
\hline Varianza total explicada & 30.63 & 28.16 & 23.01 \\
Varianza total estandarizada & 37.44 & 34.43 & 28.13 \\
\hline & Matriz para el cálculo de las puntuaciones & \\
\hline vh & .364 & -.096 & .018 \\
ATH & .347 & -.050 & -.104 \\
EH & .362 & -.167 & .049 \\
PAT & -.048 & .398 & -.095 \\
UE & .150 & .311 & -.230 \\
ICI & -.188 & .455 & -.056 \\
EC & .028 & -.075 & .396 \\
TEL & -.062 & .168 & .254 \\
CEL & -.089 & -.177 & .578 \\
\hline
\end{tabular}

Fuente: elaboración propia. 
cada variable. Las puntaciones se multiplican por cada variable para cada individuo y momento. Con esa ponderación se obtienen los valores de los factores. Mientras para el cálculo de la CI cada uno de los factores es re-ponderado por su carga factorial, de la forma siguiente:

$$
\mathrm{CI}_{\mathrm{it}}=\sum_{\mathrm{k}=1}^{K} \mathrm{~F}_{\mathrm{kit}} \beta_{k}
$$

Donde $C I$ indica la capacidad innovadora del individuo $i$ en el momento $t, F$ es el factor $k$ que representa los valores re-escalados de las variables originales y $\beta$ es la carga de cada factor dada su varianza total explicada, la cual es re-escalada de forma que $\sum_{\mathrm{k}=1}^{\mathrm{K}} \beta_{k}=1$. En este sentido, la CI es una variable que engloba la productividad de cada uno de los sistemas de innovación estatal, el cual es utilizado como base en el análisis comparativo y econométrico espacial.

En la figura 2 se presenta la distribución geográfica del PIB per cápita, las solicitudes de patentes por cada 10,000 habitantes y la CI, las cuales se obtuvieron de conacyt, Inegi y mediante la función (7) respectivamente, mientras en el cuadro 3 se presenta el resumen estadístico.

\section{Cuadro 3 \\ Resumen estadístico}

\begin{tabular}{lcccc}
\hline \multicolumn{1}{c}{ Variables } & Media & Máximo & Mínimo & Desviación estándar \\
\hline Patentes solicitadas & 0.038 & 0.264 & 0.000 & 0.048 \\
Capacidad innovadora & 5.987 & 21.522 & 0.334 & 4.385 \\
ln PIB per cápita & 11.383 & 13.992 & 10.619 & 0.590 \\
\hline
\end{tabular}

Fuente: elaboración propia.

En el caso de las solicitudes de patentes su distribución geográfica es heterogenea, solo las regiones con bajas solicitudes se encuentran aglomeradas, principalmente la región sur de México, mientras Nuevo León y la Ciudad de México se encuentran con valores atípicos superiores. En el caso de la CI presenta su distribución espacial es más homogénea y el único valor inferior atípico es Oaxaca. La frontera-norte y las regiones próximas la Ciudad de México presentan los valores promedio superiores. En el caso del PIB las regiones presentan una distribución similar a la CI salvo que los valores altos están dispersos en Baja California, Baja California Sur, Coahuila, Nuevo León, la Ciudad de México, Tabasco, Campeche y Quintana Roo, mientras el valor extremo superior se encuentra en Campeche. 


\section{Figura 2}

Distribución geográfica promedio de la capacidad innovadora y el PIB
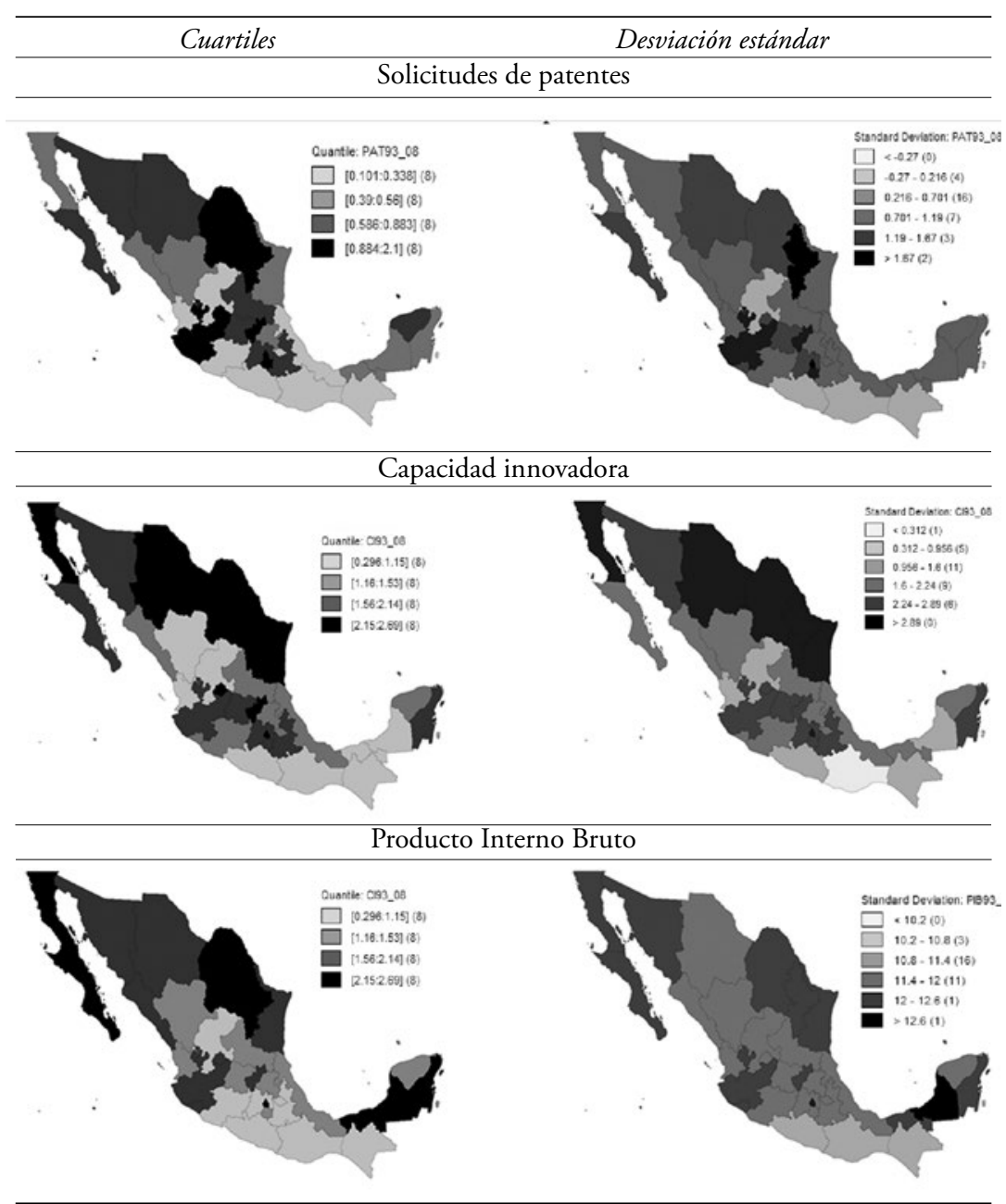

Fuente: elaboración propia. 


\section{Análisis de resultados}

En la mayor parte de la información aquí utilizada parece presentarse una cierta relación espacial entre los distintos indicadores de las entidades. En las figuras 3, 4 y 5 se presentan los casos de asociación espacial global, mediante el I de Moran (véase ecuación 3) y el scatter plot (véase figura 1), y local, mediante el indicador LISA (véase ecuación 4) en un mapa de cluster.

Con la información de los $i$ de Moran es posible presentar algunas conclusiones generales. El hecho de que todos los indicadores significativos sean positivos es un cierto indicativo de un proceso espacial de polarización debido a la separación y concentración de las entidades con sus similares. Una segunda implicación está asociada al valor del estadístico $i$ ya que al ser indicadores bajos, alejados de la unidad, 0.2 en promedio, se presenta una asociación espacial negativa débil. Un indicativo de varios trabajos de corte espacial (Rey y Sastré, 2010; Sastré y Rey, 2013 y Torres-Preciado et al., 2014) es la escasa asociación espacial global, explicada no sólo por las diferencias productivas entre las regiones sino por la distribución geográfica, los movimientos poblacionales, los medios de comunicación físicos, la densidad demográfica e incluso rasgos culturales comunes dentro de las regiones.

La información de los indicadores locales y globales son consistentes entre sí ya que la mayoría de clusters son del tipo 1 y 2, high-high y lowlow, como reflejo de asociación espacial positiva o polarización regional. Una de las cosas interesantes en torno a la clusterización es una persistente aparición de indicadores espaciales bajos en la franja sur (Guerrero, Oaxaca, Chiapas, Tabasco, Campeche, Veracruz y Yucatán). Al tomar todo el periodo y sub-periodos de análisis, la evidencia es consistente; en la franja sur se concentran los indicadores espaciales consistentemente bajos.

Un paso previo a la estimación del efecto de la capacidad para innovar en el crecimiento es realizar las pruebas para verificar la existencia de efectos espaciales. Si bien la ubicación y la interacción espacial influyen en la vida económica, el proceso de aprendizaje en el tiempo es fundamental para la acumulación de capacidades. En el cuadro 4 se presentan las estimaciones de prueba mediante una ecuación autoregresiva.

En todos los casos, la dinámica temporal es significativa y robusta, para probar de forma rigurosa el efecto espacial. En el periodo 2003-2008, referente a los efectos regionales, no hay evidencia de ninguna relación espacial al ser rechazadas todas las hipótesis de dependencia. En el periodo 1998-2002, sólo en el caso del PIB per cápita hay indicios de dependencia espacial por el i de Moran, orientándose al modelo de error espacial dada 


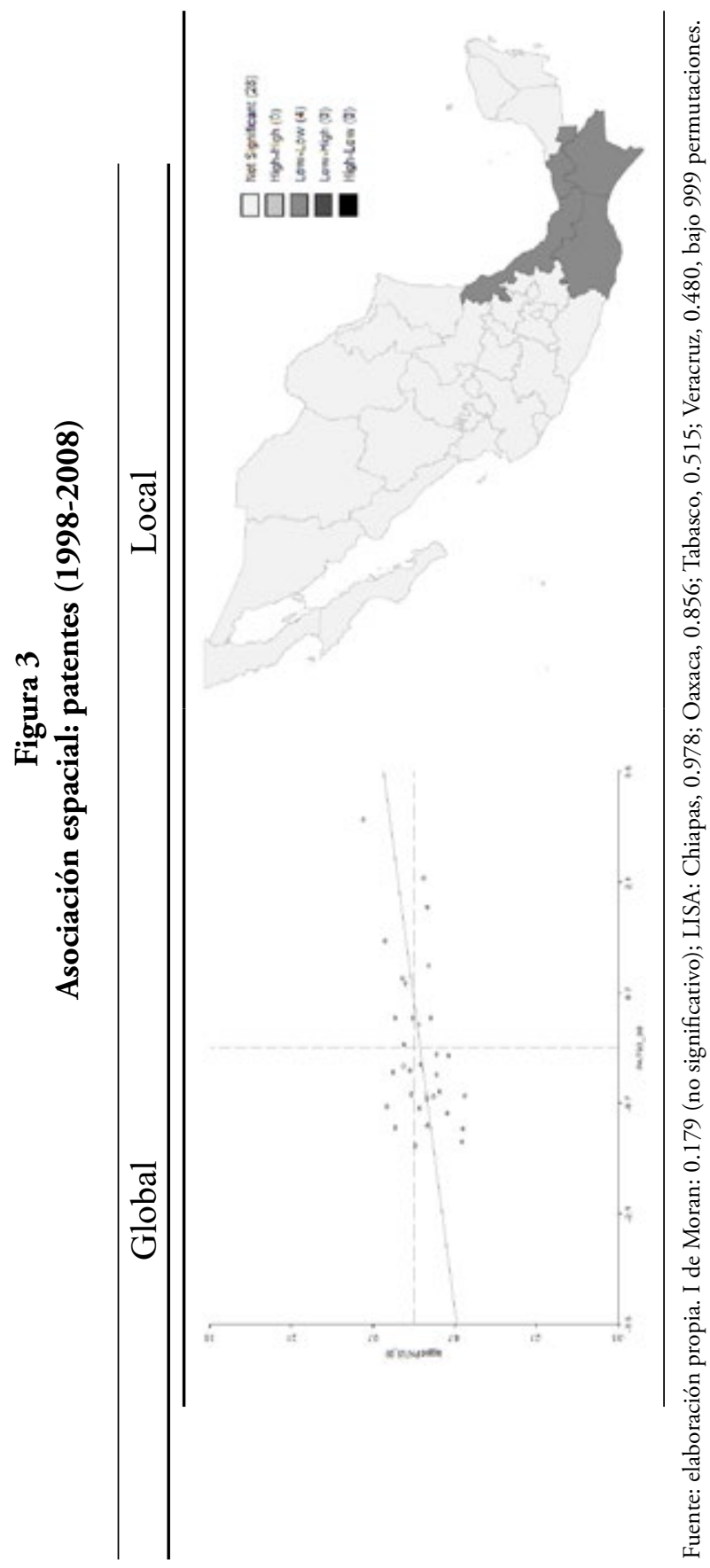




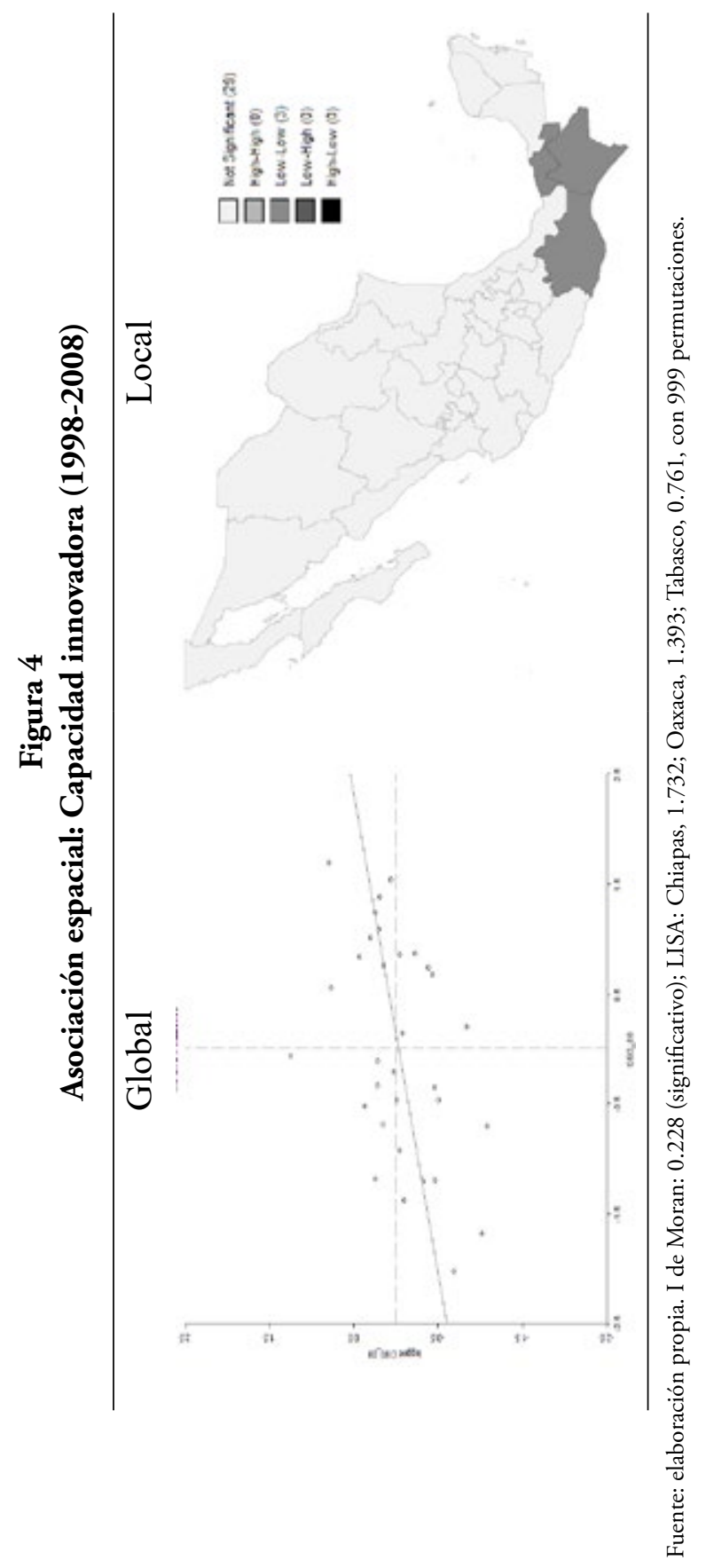




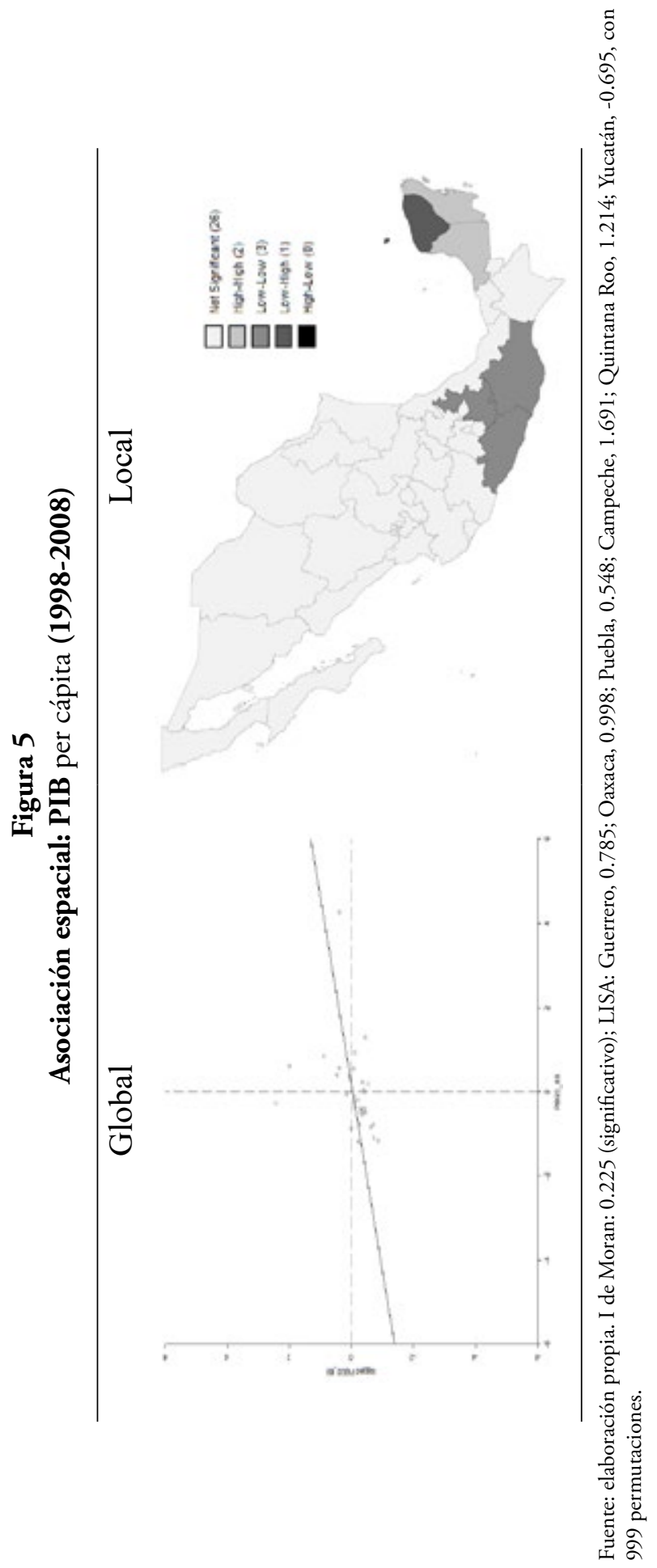




\section{Cuadro 4 \\ Estimaciones de prueba para análisis espacial}

\begin{tabular}{lcccccc}
\hline & \multicolumn{3}{c}{$2003-2008$} & \multicolumn{3}{c}{$1998-2002$} \\
\cline { 2 - 6 } & PIB & PAT & $C I$ & PIB & PAT & $C I$ \\
\hline PIB (-1) & $.9866^{*}$ & & & $.9688^{*}$ & & \\
& $(.0164)$ & & & $(.0158)$ & & \\
PAT (-1) & & $.9173^{*}$ & & & $.9114^{*}$ & \\
& & $(.0900)$ & & & $.0767)$ & \\
CI (-1) & & & $.6888^{*}$ & & & $.8315^{*}$ \\
& & & $.0250)$ & & & $(.0347)$ \\
\hline$i$ de Moran & .1718 & .1543 & .1579 & .0141 & .5160 & .8885 \\
$L M_{\text {lag }}$ & .4164 & .3232 & .9655 & .1551 & .7815 & 8728 \\
$L M_{\text {le }}$ & .4735 & .7740 & .7144 & .1039 & .9213 & .9945 \\
$L M_{\text {err }}$ & .3416 & .1022 & .3131 & .0526 & .7607 & .6753 \\
$L M_{\text {el }}$ & .3840 & .1825 & .2837 & .0363 & .8729 & .6790 \\
Sarma & .4921 & .2525 & .5623 & .0407 & .9500 & .9063 \\
\hline
\end{tabular}

Fuente: elaboración propia. Entre paréntesis se presenta el error estándar. El * representa la significancia a 5\%. En los estadísticos de prueba se presentan las probabilidades.

la significancia de los estadísticos, , y sarma. Estos resultados son similares a lo encontrado por Aroca et al. (2005) ya que presentan evidencia de que a partir de la apertura comercial en 1994 el efecto de la dependencia espacial ha disminuido progresivamente y se han conformado clusters regionales.

Con estos resultados es posible estimar las ecuaciones 1 y 2 sin sospechar de una especificación incorrecta. En el cuadro 5 se presentan las estimaciones con la especificación espacial para el periodo 1998-2002 y 2003-2008. De igual forma las estimaciones se realizan en términos logarítmicos, presentándose sólo la variable donde resultaron efectos espaciales significativos.

En el periodo 2003-2008, aunque existe un efecto espacial sustantivo de $3 \%$ en la especificación " 2 ", los efectos espaciales no son consistentes y solo la CI presenta efectos significativos de $2 \%$, por lo que el crecimiento responde casi por exclusividad a la propia dinámica temporal. En el periodo 1998-2002, el efecto espacial residual es consistente, el indicador de las patentes presenta un impacto de $5.37 \%$ y la CI de $4.1 \%$. Por una parte, el indicador tecnológico utilizado es diferenciado ya que las solicitudes de patentes presentan una mayor variación temporal y espacial, no necesariamente asociadas a la propia dinámica económica, mientras la CI muestra indicadores más estables. 


\section{Cuadro 5}

Estimaciones espaciales de ingreso e innovación

\begin{tabular}{lcccc}
\hline & \multicolumn{2}{c}{ PIB $2003-2008$} & \multicolumn{2}{c}{ PIB 1998-2002 } \\
\cline { 2 - 5 } & $(1)$ & $(2)$ & $(1)$ & $(2)$ \\
\hline PIB (-1) & $.9756^{*}$ & $.9778^{*}$ & $.9646^{*}$ & $.9699^{*}$ \\
& $(.0120)$ & $(.0109)$ & $(.0103)$ & $(.0125)$ \\
WPIB & $.0307^{*}$ & .0210 & -.0237 & -.0375 \\
\multirow{2}{*}{ AWe } & $(.0142)$ & $(.0143)$ & $(.0263)$ & $(.0329)$ \\
& .1935 & .1221 & $.3555^{* *}$ & $.4947^{*}$ \\
PAT $(-1)$ & $(.2432)$ & $(.2576)$ & $(.2133)$ & $(.2284)$ \\
& .0258 & & $.0537^{*}$ & \\
CI $(-1)$ & $.0183)$ & & $(.0140)$ & \\
& & $.0202^{* *}$ & & $.0410^{*}$ \\
\hline
\end{tabular}

Fuente: elaboración propia. Entre paréntesis se presentan los errores estándar. El * representa la significancia al $5 \%$ y el ${ }^{* *}$ la significancia al $10 \%$. wpib representa el rezago espacial del PIB per cápita y el error espacial.

Un resultado interesante es el hecho de que el efecto de la capacidad para innovar en el crecimiento ha descendido a medida que el efecto locativo ha perdido importancia. Un signo evidente con el proceso de globalización, mediante el mejoramiento de los medios de transporte y la comunicación, es la perdida de la ventaja relativa de la ubicación. Muchas de las empresas con el objetivo de incrementar su competitividad han optado por adquirir las tecnologías procedentes de las economías desarrolladas, pues adquiren tecnologías probadas y disminuyen sus costos de producción a partir de eliminar la experimentación. Estos hechos acentúan la ruptura de los encadenamientos productivos locales, reduciendo el crecimiento económico y tecnológico por la disminución de los efectos de la difusión espacial del conocimiento y todos los efectos que esto conlleva.

Al contrastar los cuadros 4 y 5 se aprecia el rechazo de la hipótesis de dependencia espacial sustantiva por una de dependencia espacial residual. En este sentido, los efectos tecnológicos y económicos de las agrupaciones regionales no surgen por efecto de la colaboración vecinal sino a algún tipo de relación locativa, ajena a las dinámicas económicas regionales. En el caso particular sucedido en el periodo 1998-2002, el rezago espacial es negativo por lo que podría argumentarse que, al menos en ese periodo, las entidades presentaban una mayor actividad de competencia que de complementariedad. 
El hecho de que el efecto espacial dominante se genere en el error es un indicativo de la ausencia de efectos espaciales directos de los vecinos, si bien ésta es una posibilidad, también pudiera ocurrir que el efecto de la dinámica propia sea lo suficientemente fuerte que el efecto del vecinal sea despreciable. Otra de las situaciones posibles está asociada a la propia distribución geográfica de las entidades, dado que las áreas urbanas, industriales o productivas en general no son un continuo en el espacio. Por ejemplo, las ciudades con mayor peso poblacional y dinamismo económico en las entidades como Tijuana (Baja California), Hermosillo (Sonora) y Ciudad Juárez (Chihuahua) no son próximas por lo que sus relaciones vecinales son más bien escasas. En este sentido, sería posible afirmar que la relación espacial entre entidades en México no es adecuada debido a que la delimitación geográfica es arbitraria y en muchos de los casos no corresponde con los límites de los fenómenos económicos y tecnológicos.

\section{Conclusiones}

Los resultados generados se orientan a una asociación espacial positiva con cluster de tipo low-low, donde los grupos de entidades con indicadores similares, especialmente bajos, se reúnen entre sí. En términos de los indicadores, la CI presenta variaciones más estables que las solicitudes de patente. Por ejemplo, en las entidades de la frontera norte, salvo el caso de Nuevo León, las solicitudes de patentes no son congruentes con sus capacidades tecnológicas, efecto que sólo es capturado por la CI. Dado los estadísticos de Moran y LISA que resultaron significativos son positivos, representan un indicativo de un proceso espacial de divergencia inter-regional, debido a la separación y concentración de los estados con sus similares. Una segunda implicación está asociada al valor del estadístico de Moran, ya que al ser indicadores bajos, alejados del rango [-1, 1] de perfecta asociación espacial, apuntan a la no complementariedad productiva e incluso a la heterogénea distribución geográfica, pues los estados del norte presentan una mayor extensión con niveles poblacionales menores y la región sur con extensión territorial pequeña y densidad demográfica mayor.

En el caso de los efectos de la capacidad para innovar en el ingreso, las solicitudes de patente presentaron efectos positivos y significativos sólo frente a la existencia de efectos espaciales en el periodo 1998-2003. En el caso de la CI, los estimadores fueron significativos en todos los casos, precisando que sus efectos fueron mayores en caso de efectos espaciales, similar al caso de las solicitudes de patentes. En el periodo posterior a la liberalización comercial el efecto espacial predominante es el del error espacial o el efecto de ubicación potencializando los efectos de la capa- 
cidad para innovar, mientras que una vez que la integración al mercado internacional se intensificaba el efecto de la ubicación desaparece, apareciendo de forma débil el efecto rezago espacial o efecto vecino donde ambas variables de innovación pierden su efecto.

Una de las cuestiones interesantes que resaltan de este análisis, bajo un marco tiempo-espacio, es el efecto de las agrupaciones geográficas sobre los efectos de la innovación en el crecimiento. Por una parte, resalta la idea de las políticas tecnológicas y económicas asociada a los sistemas nacionales y regionales de innovación y sus alcances inter-regionales como mecanismos generadores de redes productivas que estimulen el crecimiento mediante los spillovers tecnológicos y económicos. Dados los resultados aquí presentados, para futuras líneas de investigación, sería interesante profundizar en los efectos que desempeñan las agrupaciones espaciales en las dinámicas productivas, particularmente por los efectos generados por la crisis mundial de 2008 en las estructuras productivas internacionales, dado que las políticas implementadas en el mundo se enfocaron en fortalecer las relaciones empresariales locales.

\section{Fuentes consultadas}

Aboites, Jaime y Manuel Soria (2008), Economía del conocimiento y propiedad intelectual. Lecciones para la economía mexicana, Siglo XXI-UAM-Unidad Xochimilco, Ciudad de México, México.

Acs, Zoltan, Luc Anselin y Attila Varga (2002), "Patents and innovation counts as a measures of regional production of new knowledge", Research Policy, 31 (7), Elsevier B.V., Amsterdam, Holanda, pp. 1069-1085.

Aghion, Phillipe y Peter Howitts (1992), "A model of growth through creative destruction", Econometrica, 60 (2), The Econometric Society, Nueva York, Estados Unidos de América, pp. 323-351.

Albornoz, Mario (2009), "Indicadores de innovación: las dificultades de un concepto en evolución", Revista Iberoamericana de Ciencia, tecnología y Sociedad, 13 (5), Organización de Estados Iberoamericanos, Buenos Aires, Argentina, pp. 9-25.

Anselin, Luc (1995), "Local indicator of spatial association-LIsA", Geographical Analysis, 27 (2), Ohio State University, Ohio, Columbus, Estados Unidos de América, pp. 99-115. 
Anselin, Luc (1988), Spatial econometrics: methods and models, Kluwer Academic Publisher, Dordrecht, Holanda.

Anselin, Luc, Attila Varga y Zoltan Acs (2000), “Geographic and sectoral characteristics of academic knowledge externalities", Papers in Regional Science, 79 (4), Springer, Amsterdam, Holanda, pp. 435-443.

Anselin, Luc, Attila Varga y Zoltan Acs (1997), "Local Geographic Spillovers between University Research and High Technology Innovations", Journal of Urban Economics, 42 (3), Elsevier B.V., Amsterdam, Holanda, pp. 422-448.

Archibugui, Daniele y Jonathan Michie (1997), Technology globalisation and economic performance, Cambridge University, Cambridge, Reino Unido.

Aroca, Patricio, Mariano Bosch y William Maloney (2005), "Spatial dimensions of trade liberalization and economic convergence: México 1985-2002", The World Bank of Economic Review, 19 (3), Oxford University Prees, Oxford, Inglaterra, pp. 345-378.

Arrow, Kenneth Joseph (1962), "The Economic implications of learning by doing", Review of Economics Studies, 29 (3), Stockholm University, Estocolmo, Suecia, pp. 155-173.

Autio, Erkko, Martin Kenney, Phillippe Mustar, Dan Siegel y Mike Wright (2014), "Entrepeneurial innovation: the importance of context", Research Policy, 43 (10), Elsevier B.V., Amsterdam, Holanda, pp. 1097-1108.

Baldwin, Richard y Toshihiro Okubo (2006), "Heterogeneous firms, agglomerations and economic geography: spatial selection and sorting", Journal of Economic Geography, 6 (3), Oxford University Press, Oxford, Inglaterra, pp. 323-346.

Bera, Anil y Mann Yoon (1992), Simple diagnostic test for spatial dependence, University of Illinois, Department of Economics (mimeo), Chicago, Estados Unidos de América.

Burridge, Peter (1980), "On the Cliff-Ord test for spatial correlation", Journal of the Royal Statistical Society Series B-Statical Methodolo- 
gy, 1 (42), John Wiley and Sons, Inc., Nueva York, Estados Unidos de América, pp. 107-108.

Butler, Joseph (1994), Geografía económica. Aspectos espaciales y ecológicos de la actividad económica, Limusa, Noriega editores, Ciudad de México, México.

Casas, Rosalba (2001), La formación de redes de conocimiento. Una perspectiva regional desde México, Universidad Nacional Autónoma de México/Anthropos, Ciudad de México, México.

Cliff, Andrew y Keith Ord (1981), Spatial process. Models and applications, Pion, Londres, Inglaterra.

Coe, David y Elhanan Helpman (1995), "International R\&D spillovers", European Economic Review, 39 (5), Elsevier B.V., Amsterdam, Holanda, pp. 859-887.

Cooke, Philip (1992), "Regional innovation systems: competitive regulation in the new Europe", Geoforum, 23 (3), Elsevier B.V., Amsterdam, Holanda, pp. 365-382.

Dosi, Giovanni, Patrick Llerena y Mauro Sylos Labini (2006), “The relation between science, technologies and their industrial explotation: an illustration through the myths and realities of the socalled 'European Paradox'”, Research Policy, 35 (10), Elsevier B.V., Amsterdam, Holanda, pp. 1450-1464.

Dosi, Giovanni (1988), "Sources, procedures, and microeconomic effects of innovation", Journal of Economic Literature, 26 (3), American Economic Association, Tennessee, Estados Unidos de América, pp. 1120-1171.

Fagerberg, Jan, Martin Srholec y Bart Verspagen (2010), “Innovation and economic development", en Browyn H. Hall y Nathan Rosenberg (eds.), Handbook of economics of innovation, vol. 2, Elsevier B. V., Amsterdam, Holanda, pp. 833-872.

Fisher, Manfred y Attila Varga (2003), "Spatial knowledge spillovers and university research: evidence from Austria", The Annals of Regional Science, 37 (2), Springer, Amsterdam Holanda, pp. 303-322. 
Fujita, Masahisa y Paul Krugman (2004), “The new economic geography: past, present and future", Papers in Regional Science, 83 (1), Springer, Amsterdam Holanda, pp. 139-164.

Furman, Jeffrey, Michael Porter y Scott Stern (2002), “The determinants of national innovative capacity”, Research Policy, 31 (6), Elsevier B.V., Amsterdam, Holanda, pp. 899-933.

Gould, David y William Gruben (1995), "El papel de los derechos de propiedad intelectual en el crecimiento económico", Economía Mexicana. Nueva Época, 4 (2), Centro de Investigación y Docencia Económica, Ciudad de México, México, pp. 265-299.

Guzmán, Alenka, Francisco López-Herrera y Francisco Venegas-Martínez (2008), "Un análisis de cointegración entre patentes y crecimiento económico en México, 1980-2008”, Investigación Económica, 71 (281), Universidad Nacional Autónoma de México, Ciudad de México, México, pp. 83-115.

Gluckler, Johannes (2007), "Economic geography and the evolution of the networks", Journal of Economic Geography, 7 (5), Oxford University Press, Oxford, Inglaterra, pp. 619-634.

Griliches, Zvi (1960), "Hybrid corn and the economics of innovation", Science, 132 (3422), American Association for the Advancement, Washington, Estados Unidos de América, pp. 275-280.

Grossman, Gene y Elhanan Helpman (1991), Innovation and growth in the global economy, The MIT Press, Cambridge, Inglaterra.

Hall, Bronwyn y Nathan Rosenberg (2010), Handbook of the economics of innovation, Elsevier, Amsterdam, Holanda.

Hernández, Sergio y Eliseo Díaz (2007), "La producción y el uso del conocimiento en México y su impacto en la innovación: análisis regional de las patentes solicitadas", Análisis Económico, 22 (50), Universidad Autónoma Metropolita-Azcapotzalco, Ciudad de México, México, pp. 185-217.

Hirschman, Albert (1958), The strategy of economic development, Yale University Press, New Haven, Inglaterra. 
Jaffe Adam y Manuel Trajtenberg (2002), Patents, citations \& innovations. A window on the knowledge economy, The MIT Press, Cambridge, Inglaterra.

Jaffe, Adam (1989), "Real effects of academic research", American Economic Review, 79 (5), American Economic Association, Tennessee, Estados Unidos de América, pp. 957-970.

Kaldor, Nicholas (1970), “The case for regional policies", Scottish Journal of Political Economy, 17 (3), John Wiley and Sons, Inc., Nueva York, Estados Unidos de América, pp. 337-348.

Krammer, Marius y Sebastian Sorin (2009), "Drivers of national innovation in transition: evidence from a panel of Eastern European countries", Research Policy, 31 (6), Elsevier B.V., Amsterdam, Holanda, pp. 899-933.

Krugman, Paul (1998), "Space: the final frontier", Journal of Economic Perspective, 12 (2), American Economic Association, Nueva York, Estados Unidos de América, pp. 161-174.

Krugman, Paul (1991), Geography and trade, The MIT Press, Cambridge, Inglaterra.

Lall, Sanjaya (1992), "Technological Capabilities and Industrialization”, World Development, 20 (2), Elsevier B.V., Amsterdam, Holanda, pp. 165-186.

LeSage, James y Kelley Pace (2009), Introduction to spatial econometrics, Chapman \& Hall/CRC, Florida, Estados Unidos de América.

López, Isabel (2008), "Inventores prolificos, conocimiento tecnológico y patentes: México y Corea”, Economía: Teoría y Práctica, 29 (2), Universidad Autónoma Metropolitana-Iztapalapa, Ciudad de México, México, pp. 87-118.

López-Leyva, Santos, Miriam Castillo-Arce, José Ledezma-Torres y Jesús Ríos-Flores (2014), "Economic growth from a theoretical perspective of knowledge economy: an empirical analysis for Mexico", Management Dynamics in the Knowledge Economy, 2 (2), Research Papers in Economics, St. Louis, Missouri, Estados Unidos de América, pp. 217-239. 
Lundvall, Bengt-Ake (1992), National systems of innovation towards a theory of innovation and interactive learning, Pinter Publishers, Londres, Inglaterra.

Mansfield, Edwin (1961), "Technical change and the rate of imitation", Econometrica, 29 (4), The Econometric Society, Nueva York, Estados Unidos de América, pp. 741-766.

Marroquin, Juan y Humberto Ríos (2012), "Inversión en investigación y crecimiento economic: un análisis desde la perspective de los modelos de I+D”, Investigación Económica, 71 (282), Universidad Nacional Autónoma de México, Ciudad de México, México, pp. 15-33.

Metcalfe, John Stanley (2002), "Knowledge of growth and the growth of knowledge", Journal of Evolutionary Economics, 12 (1), Springer, Amsterdam Holanda, pp. 3-15.

Mendoza, Jorge, Víctor Torres y Mayrén Polanco (2008), “Desigualdad del crecimiento económico regional e innovación tecnológica en México", Comercio Exterior, 58 (7), BANCOMEXT, Ciudad de México, México, pp. 507-521.

Moran, P. (1948), "The interpretation of statistical maps", Journal of the Royal Statistical Society, B, 10 (2), John Wiley and Sons, Inc., Nueva York, Estados Unidos de América, pp. 243-251.

Moreno, Rosina y Esther Vayá (2002), "Econometría espacial: nuevas técnicas para el análisis regional. Una aplicación a las regiones europeas", Investigaciones regionales, núm. 1, Asociación Española de Ciencia Regional, Madrid, España. pp. 83-106.

Moreno, Rosina y Esther Vayá (2000), Técnicas econométricas para el tratamiento de datos espaciales: la econometría espacial, Universitat de Barcelona, Barcelona, España.

Mungaray, Alejandro y Juan Ignacio Palacio (2000), "Schumpeter, la innovación y la política industrial”, Comercio Exterior, 50 (12), BANCOMEXT, Ciudad de México, México, pp. 1085-1089.

Myrdal, Gunnar (1957), Economic theory and underdevelopment regions, University Paperbacks, Methuen, Londres, Inglaterra. 
Nelson, Richard (1994), "The co-evolution of technology, industrial structure and supporting institutions", Journal Industrial and Corporate Change, 3 (1), Oxford University Prees, Oxford, Inglaterra, pp. 47-63.

Nelson, Richard y Sidney Winter (1982), An evolutionary theory of economic change, Belknap Press-Harvard University Press, Cambridge, Inglaterra.

Ocegueda, Juan Manuel, Ramón Castillo y Rogelio Varela (2009), "Crecimiento regional en México: especialización y sectores clave", Problemas del Desarrollo. Revista Latinoamericana de Economía, 40 (159), Universidad Nacional Autónoma de México, Ciudad de México, México, pp. 61-84.

Omidvar, Omid (2013), "Revisiting absorptive capacity: literature review and a practice-based extension of the concept", 35th DRUID Celebration Conference, 17-19 de junio, Barcelona, España, $<$ http://druid8.sit.aau.dk/acc_papers/ticbvh43b3s856q09059tnbrv726.pdf $>, 20$ de febrero de 2015.

Oster, Scharon (2000), Análisis moderno de la competitividad, Oxford University Press, Oxford, Inglaterra.

Pavitt, Keith (1984), "Sectoral patterns of technical change: towars and taxonomy and a theory", Research Policy, 13 (6), Elsevier B.V., Amsterdam, Holanda, pp. 343-373.

Pérez, César (2006), Análisis Multivariante con aplicaciones para SPSS 15, Prentice Hall, New Jersey, Estados Unidos de América.

Porter, Michael (1990), The Competitive Advantage of Nations, Free Press, Nueva York, Estados Unidos de América.

Rey, Sergio (2000), "Integrated regional econometrics +input -output modeling: issues and opportunities", Papers in Regional Science, 79 (3), Springer, Amsterdam Holanda, pp. 271-292.

Rey, Sergio y Brett Mounturi (1999), "US regional income convergence: a spatial econometrics perspective", Regional Studies, 33 (2), Taylor and Francis Group, Londres, Inglaterra, pp. 143-156. 
Rey, Sergio y Myrna Sastré (2010), "Interregional inequality dynamics in Mexico”, Spatial Economic Analysis, 5 (3), Regional Studies Association, Londres, Inglaterra, pp. 277-298.

RICYT (Red de Indicadores de Ciencia y Tecnología) (2001), Normalización de indicadores de innovación tecnológica en América Latina y el Caribe Manual de Bogotá, RICYT/OEA/CYTED/ COLCIENCIAS/OYT, Bogotá, Colombia, < http://www.ricyt. org/manuales/doc_view/5-manual-de-bogota $>$, 15 de octubre de 2013.

Ríos, Jesús y Miriam Castillo (2015) "Efectos de la capacidad innovadora en el crecimiento económico. Análisis comparativo entre países desarrollados y en desarrollo", Región y Sociedad, 27 (64), El Colegio de Sonora, Hermosillo, México, pp. 109-138.

Ríos, Humberto y Juan Marroquín (2013), “Innovación tecnológica como mecanismo para impulsar el crecimiento económico. Evidencia regional para México", Contaduría y Administración, 58 (3), Universidad Nacional Autónoma de México, Ciudad de México, México, pp. 11-37.

Ríos, Jesús y Miriam Castillo (2013), "La capacidad innovadora y el desempeño económico y tecnológico de México", en Juan Carlos Bermúdez (ed.), Emprendimiento e innovación para la competitividad internacional. Dimensiones, factores y esquemas empiricos sobre sus causas y efectos, Escuela de Relaciones Internacionales de la Universidad Nacional Costa Rica, Heredia, Costa Rica, pp. 41-76.

Romer, Paul (1990), "Endogenous technological change", Journal of Political Economy, 98 (5), The Univesity of Chicago Prees, Chicago, Estados Unidos de América, pp. 71-102.

Rozga, Riszard (2008), "Algunos modelos territoriales de innovación y su aplicación en México", en Corona, Leonel y Santos López (Coord), Innovación y conocimiento. Dinámicas regionales en el noroeste de México, RIDIT, UAS, Sinaloa, México.

Ruiz, Clemente (2008), "México: geografía económica de la innovación”, Comercio Exterior, 58 (11), BANCOMEXT, Ciudad de México, México, pp. 756-768. 
Sala-i-Martin, Xavier (2000), Apuntes de crecimiento económico, Antoni Bosch, Barcelona, España.

Sánchez, Yesenia, Francisco García y Esteban Mendoza (2014), “Determinantes de la capacidad innovadora regional en México. Una tipología de las regiones" Región y Sociedad, 26 (61), el Colegio de Sonora, Hermosillo, México, pp. 119-160.

Sastré, Myrna y Sergio Rey (2013), "Space-time income distribution dynamics in Mexico", The Annals of GIS, 19 (3), Taylor and Francis Group, Londres, Inglaterra, pp. 195-207.

Solo, Robert (1966), "The capacity to assimilate an advanced technology”, American Economic Review, 56 (1/2), American Economic Association, Tennessee, Estados Unidos de América, pp. 91-97.

Solow, Robert (1957), "Technical change and the aggregate production function", The Review of Economics and Statistics, 39 (3), The MIT Press, Cambridge, Inglaterra, pp. 312-320.

Torres-Preciado, Víctor, Mayrén Polanco-Gaytan y Miguel TinocoZermeño (2014), "Technological innovation and regional economic growth in Mexico: a spatial perspective", The Annals of Regional Science, 52 (1), Springer, Amsterdam, Holanda, pp. 183-200.

Valdivia, Marcos (2007), "Heterogeneidad espacial, convergencia y crecimiento regional en México", ponencia presentada en XVII Coloquio de Economía Matemática y Econometría, 21-25 de mayo, Quintana Roo, México.

Valdez-Lafarga, Cuitláhuac y Jorge León-Balderrama (2015), "Hacia una taxonomía de los sistemas regionales de innovación en México", Economía, Sociedad y Territorio, 15 (48), El Colegio Mexiquense A. C., Toluca, Estado de México, pp. 517-553.

Vázquez-Barquero, Antonio (2005), Las nuevas fuerzas del desarrollo, Antoni Bosch, Barcelona, España.

Villareal, Edna (2012) "Innovación y crecimiento regional en México 2000-2010”, ponencia presentada en Congreso de la Asociación Española de Ciencia Regional, XXXVIII Reunión de Estudios Regionales, 22-23 noviembre, Bilbao, España. 
Recibido: 1 de octubre de 2015. Corregido: 26 de marzo de 2016. Aceptado: 27 de enero de 2017.

Jesús Armando Ríos-Flores. Doctor en ciencias económicas por la Universidad Autónoma de Baja California, actualmente es profesor de la Facultad de Ciencias Sociales y Políticas de la misma institución. Es miembro del Sistema Nacional de Investigadores nivel "c", con la línea de investigación en innovación, competitividad y crecimiento. Entre sus últimas publicaciones destacan: "Efectos de la capacidad innovadora en el crecimiento económico. Análisis comparativo entre países desarrollados y en desarrollo", Región y Sociedad, 27 (64), El Colegio de Sonora, Hermosillo, México, pp. 109-138 (2015); "La capacidad de absorción tecnológica en el crecimiento económico", Revista Iberoamericana de Ciencia, Tecnología y Sociedad, 12 (34), Centro de estudios sobre Ciencias, Desarrollo y Educación Superior, Buenos Aires, Argentina, pp. 197-222 (2017); en coautoría: "Knowledge activities in mexican states: a territorial visión", en Alexandra Zbuchea, Constantin Bratianu y Florina Pinzaru (eds.), Economic behavior: economy, business \& people, Cambridge Scholars Publishing, Londres, Inglaterra, pp. 48-84 (2017).

Juan Manuel Ocegueda-Hernández. Doctor en economía por la Universidad Nacional Autónoma de México. Actualmente es profesor de Desarrollo Económico y Macroeconomía del Programa de Maestría y Doctorado en Ciencias Económicas en la Facultad de Economía y Relaciones Internacionales de la Universidad Autónoma de Baja California y Rector de la misma institución. Es miembro del Sistema Nacional de Investigadores nivel 2. Sus líneas de investigación son crecimiento económico y desarrollo regional, y Educación superior y desarrollo. Entre sus últimas publicaciones destacan: "Análisis comparado de la cobertura de la educación superior en Corea del Sur y Chile: una reflexión para México", Perfiles Educativos, XXXIX (155), Universidad Nacional Autónoma de México, Ciudad de México, México, pp. 141-159 (2017), "Labor Informality in Mexico: An Indicator Analysis", en Abel Polese, Colin Williams, Loana Horodnic y Predrag Bejakovic (eds.), The Informal Economy in Global Perspective. Varieties of Governance, Palgrave-MacMillan, International Political Economy Series, Londres, Inglaterra, pp. 197-214 (2017) y como coordinador en La responsabilidad social de la universidad mexicana a mitad del siglo XXI, Universidad Autónoma de Baja CaliforniaMiguel Ángel Porrúa, Mexicali, México (2016). 